\title{
Efecto de la variedad de orujo de uva sobre la producción de gas y metano in vitro
}

\author{
Barraso, $C^{1}$.; López-Parra, M.Mํ. and Rodríguez, P.L².
}

'Departamento de Calidad de Carnes. Centro de Investigaciones Científicas y Tecnológicas de Extremadura (CICYTEX). Badajoz. España. ${ }^{2}$ Departamento de Producción Animal y Ciencia de los Alimentos. Facultad de Veterinaria. Universidad de Extremadura. Cáceres. España.

PaLABRAS ClaVe

Fermentación in vitro.

Orujos de uva.

Producción de gas.

Producción de metano.

\section{SUMMARY}

El metano es un gas de efecto invernadero que contribuye potencialmente al cambio climático. Los rumiantes, debido a las características de su sistema digestivo, son capaces de digerir la fibra mediante el proceso de fermentación ruminal, en el cuál se generan gases como el metano. Los polifenoles presentan capacidad para inhibir la producción de dicho gas. Estos compuestos están presentes en distintas plantas y subproductos agroindustriales, como el orujo de uva, cuya inclusión en la dieta de corderos podría dar lugar a una disminución de las emisiones de metano entérico. Por tanto, el objetivo de este estudio, ha sido evaluar la cinética de producción de gas, así cómo la producción de metano de 15 variedades de orujos de uva (blancos y tintos), con el fin de seleccionar aquellas variedades que sean menos metanogénicas y poder incorporarlas en el pienso de cebo de corderos. Para ello, se ha empleado la técnica de producción de gas in vitro en dos periodos de fermentación diferentes, 18 y 96 horas. Los resultados obtenidos para la producción de gas a 18 y 96 horas en los orujos tintos han sido de $59.77-104.28 \mathrm{ml} / \mathrm{g}$ y para los blancos $94.44-173.23 \mathrm{ml} / \mathrm{g}$ respectivamente. En cuanto a la producción de metano en orujos tintos obtuvimos 3.72-6.79 $\mathrm{mlCH}_{4} / \mathrm{g}$ frente a $9.66-26.08 \mathrm{mlCH}_{4} / \mathrm{g}$ producidos por los orujos blancos. Por tanto, se observa un mayor potencial inhibidor de la producción de gas y metano por parte del grupo de orujos tintos frente al de los orujos blancos.

\section{In vitro gas and methane production by grape pomaces, influency of variety}

\section{RESUMEN}

\section{ADDITIONAL KEYWORDS}

Gas production.

Grape pomaces.

In vitro fermentation.

Methane production.

\section{INFORMATION}

Cronología del artículo.

Recibido/Received: 16.04 .2020

Aceptado/Accepted: 02.11.2020

On-line: 15.01.2021

Correspondencia a los autores/Contact e-mail:

carmen.barraso@juntaex.es.
Methane is a greenhouse gas that potentially contributes to climate change. The characteristics of the digestive system of ruminants enable them to digest fibre by ruminal fermentation, generating gases such as methane. Polyphenols have the ability to inhibit the production of this gas. These compounds are available in different plants and agro-industrial by-products, such as grape pomaces, whose inclusion in diet of lambs could cause a decrease in enteric methane emissions. Therefore, the aim of this study was to evaluate the kinetics of gas production, as well as the production of methane from 15 varieties of grape pomace (white and red), in order to select those varieties with less methanogenic characteristics and include them in lamb feed. To this purpose, the in vitro gas production technique has been used in two different fermentation periods, 18 and 96 hours. The results obtained for gas production at 18 and 96 hours in red pomaces were $59.77-104.28 \mathrm{ml} / \mathrm{g}$ and for white pomace $94.44-173.23 \mathrm{ml} / \mathrm{g}$ respectively. In terms of methane production in red pomaces we obtained 3.72-6.79 $\mathrm{mlCH}_{4} / \mathrm{g}$ compared to $9.66-26.08 \mathrm{mlCH}_{4} / \mathrm{g}$ produced by white pomaces.

\section{INTRODUCCIÓN}

La reducción de los gases de efecto invernadero (GEI) es un requisito internacional en la actualidad, debido a que han sido reconocidos como responsables significativos del calentamiento global del planeta (Ramanathan \& Feng 2009). Dentro de este grupo de gases, encontramos el metano $\left(\mathrm{CH}_{4}\right)$, que presenta un potencial de calentamiento 25 veces mayor al dióxido de carbono $\left(\mathrm{CO}_{2}\right)$ (IPCC, 2007). Según informes de la
FAO (Steinfeld et al. 2006) los rumiantes, son responsables del 30\% de las emisiones de $\mathrm{CH}_{4}$ asociadas a la producción de alimento de origen animal. $\mathrm{El} \mathrm{CH}_{4}$ es un subproducto de la fermentación de la materia orgánica en el rumen y es responsable de la pérdida de entre $2-12 \%$ de la energía bruta proporcionada por la dieta (Hristov et al. 2015; Moumen et al. 2016). Este gas, es resultante de la fermentación anaerobia de un complejo sistema simbiótico entre bacterias, hongos y protozoos, capaces de digerir el material fibroso y convertirlo en 
ácidos grasos volátiles (AGV), gases de fermentación y calor (Alejos et al. 2018). El exceso de $\mathrm{H}_{2}$ generado en la conversión de glucosa a $\mathrm{AGV}$ y el $\mathrm{CO}_{2}$ producido por la descarboxilación de metabolitos durante la fermentación, son utilizados como sustratos por las arqueas y protozoos, para transformarlos en $\mathrm{CH}_{4}$ (Hook et al. 2010; Alayon et al. 2018).

Existen planteadas distintas estrategias para la mitigación de la producción de $\mathrm{CH}_{4}$ ruminal, entre ellas, la incorporación de aditivos químicos en la alimentación del ganado, sin embargo, esta medida se ve limitada por la posible aparición de residuos en productos de origen animal para consumo humano, lo cual, lleva a ciertas restricciones legales como la retirada del uso de antibióticos, entre ellos la monensina, en la Unión Europea desde enero de 2006 (EC 2003). Por ello, existe una continua búsqueda de compuestos bioactivos naturales, como alternativa al uso de químicos. Estos compuestos están presentes en plantas y subproductos agroindustriales, como es el caso de los taninos, pertenecientes al grupo de los polifenoles. Estos, presentan capacidad inhibitoria sobre la producción del $\mathrm{CH}_{4}$ a nivel ruminal, debido a sus propiedades antimicrobianas (Patra \& Saxena 2010; Broucek 2018), ya que actúan sobre la pared celular y los enzimas extracelulares, inhibiendo el transporte de nutrientes y dificultando el crecimiento microbiano (McSweeney et al. 2001).

Dentro del grupo de subproductos agroindustriales destaca el orujo de uva, resultante del proceso de elaboración de diferentes vinos y caracterizado por presentar un alto contenido en polifenoles (Peixoto et al. 2018). Su inclusión en la dieta de corderos supondría un sistema sinérgico entre agricultura y ganadería, disminuyendo por una parte, el exceso de biomasa generado por la industria vitivinícola y, por otra parte, reduciendo la emisión de GEI procedente de los rumiantes, en concreto de $\mathrm{CH}_{4}$ ruminal, dando lugar a una producción de corderos más sostenible y respetuosa con el medio ambiente.

Por lo tanto, el objetivo de este trabajo fue analizar 15 variedades de orujo de uva (blancos y tintos), de las cuales, se realizó un estudio de la producción de $\mathrm{CH}_{4}$ y gas total tras 18 horas de fermentación, así como, un estudio de la cinética de producción de gas y la producción de $\mathrm{CH}_{4}$ tras 96 horas de fermentación in vitro en un sistema de jeringas, con la finalidad de determinar la influencia varietal (blanca y tinta) sobre estos parámetros y detectar variedades con mayor potencial antimetanogénico. El objetivo último es incorporar estas variedades en el concentrado de cebo de corderos para tratar de disminuir la producción de $\mathrm{CH}_{4}$ durante su crecimiento.

\section{MATERIAL Y MÉTODOS}

Se han utilizado 15 variedades distintas de orujo de uva procedentes de la comarca de Almendralejo (Badajoz, Suroeste de España), 8 tintas y 7 blancas, de la campaña 2018. Tras su recepción en el laboratorio, las muestras fueron desecadas en estufa para determinar su contenido en humedad y facilitar su molienda en molino ciclónico con tamiz de 1mm (Rescht ZM200). Los análisis se han realizado siguiendo el Reglamento
CE 152/2009 (EC 2009), para cenizas, materia orgánica (M.O), grasa bruta (G.B), proteína bruta (P.B), fibra bruta (F.B), fibra neutro detergente (F.N.D) y fibra ácido detergente (F.A.D).

El proceso de fermentación in vitro ha seguido básicamente la técnica propuesta por Menke et al. (1979) utilizando jeringas de vidrio de $120 \mathrm{ml}$ de capacidad con punta tipo Luer y midiendo el desplazamiento del émbolo producido por el gas de fermentación. La punta Luer se cierra con una válvula de tres vías que garantiza un cierre hermético y permite acoplar distintos dispositivos de medida.

Como fuente de inóculo para la fermentación, se utilizaron heces recogidas directamente del recto de ovejas (El Shaer, Omed \& Chamberlain. 1987) pertenecientes a la granja de la Facultad de Veterinaria de Cáceres y finca la Orden-Valdesequera, inmediatamente antes del inicio del proceso. El procedimiento consta de los siguientes pasos:

Fabricación de saliva artificial a partir de cuatro soluciones (Aghajanzadeh et al. 2015): solución macromineral, solución tampón, solución reductora y solución micromineral.

Para iniciar el proceso de fermentación depositamos $0,2 \mathrm{~g}$ de orujo de estudio junto con $30 \mathrm{ml}$ de líquido fecal (mezcla homogénea de heces y saliva artificial) en una jeringuilla y posteriormente se incuban en una estufa a una temperatura de $39^{\circ} \mathrm{C}$.

En cada ensayo se emplearon dos juegos de 12 jeringas, de las cuales, dos actúan de blanco $(30 \mathrm{ml}$ de líquido fecal), otras dos actúan de testigo ( $0.2 \mathrm{~g}$ de cebada como sustrato fermentador de referencia), las ocho restantes contienen como sustrato fermentador los orujos de estudio (8/variedad). El proceso de fermentación se desarrolló en dos condiciones temporales diferentes, por una parte, se midió la producción potencial de gas y $\mathrm{CH}_{4}$ tras 18 horas de incubación ya que se considera suficiente para determinar el potencial de degradación de un concentrado (Damiran \& Yu 2010) y por otra parte, las mismas muestras se sometieron a un proceso de incubación de 96 horas, anotando el avance del émbolo a las $0,2,4,6,8,10,12,24,48,72$ y 96 horas siguiendo el protocolo ordinario (El Meadaway et al. 1998; Rasoul \& Amiri 2016), con el fin de estudiar la cinética de producción de gases y la formación de $\mathrm{CH}_{4}$ tras 96 horas de fermentación. Las curvas de producción de gas se modelizaron siguiendo los modelos propuestos por Orskov \& McDonald (1979) $p=a+b\left(1-e^{-c t}\right)$ y McDonald (1981) $p=a+b\left(1-e^{-c(t-t l)}\right)$.

La medida de la producción de $\mathrm{CH}_{4}$ se realizó al terminar la prueba de fermentación a las 18 y 96 horas, mediante un detector de gases GMI PS211. Este detector determina el porcentaje de $\mathrm{CH}_{4}$ en el gas producido utilizando un sistema de circuito cerrado de gases conducidos a un bote Duran de 250-500ml para las jeringas incubadas a 18 y 96 horas respectivamente,

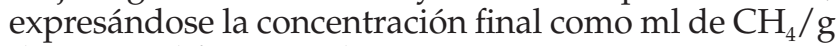
de material fermentado.

El posible efecto de la variedad de orujo de uva (tinto/blanco) sobre la producción de gas total y $\mathrm{CH}_{4}$ se estudió mediante un análisis prueba de Kruskall- 
Wallis para establecer las posibles diferencias estadísticamente significativas ( $\mathrm{p}<0.05)$, para ello se utilizó el programa SPSS (V22). La modelización de la cinética de producción de gas, se ajustó mediante programación Solver, en Excel, minimizando la diferencia de cuadrados entre los datos reales y ajustados al variar las constantes de la ecuación $(a, b, c, t l)$.

\section{RESULTADOS Y DISCUSIÓN}

En la Tabla I se recogen los datos referentes a la composición bromatológica de los orujos de estudio. Podemos observar que no existen diferencias significativas en ninguno de los componentes analizados para las variedades tintas y blancas. Podríamos definir nutritivamente este subproducto como fibroso, con un contenido moderado en proteínas y ligeramente alto en grasas. Los valores obtenidos para la composición química de los orujos, se encuentran en el rango de variabilidad descrito por diferentes autores (Baumgärtel et al. 2007; Abarghuei, Rouzbehan \& Alipour. 2010; Moate et al. 2014; Foiklan, Wanapat \& Norrapoke. 2016). Esta variabilidad, puede ser debida a que los componentes que lo forman (hollejo, granilla y pulpa) no siempre se encuentran en la misma proporción, debido tanto al procesado de la uva como a la variedad vegetal utilizada (Deng, Penner \& Zhao. 2011). La G.B es aportada principalmente por la granilla (Marginet 2004). La escasa diferencia entre F.N.D y F.A.D y el elevado valor de esta última, presupone una baja fermentabilidad de la fracción fibrosa (McDonald et al. 2010; Van Soest 1967).

En la Figura 1 y Tabla III se encuentran representados los valores de producción de gas tras 96 horas de fermentación. Gráficamente se puede observar que los orujos blancos empiezan a fermentar con mayor potencia que los tintos y con una producción mayor de gas desde el inicio hasta el final del proceso de fermentación. El mismo comportamiento vemos en fermentaciones a 18 horas (Tabla II). Son los orujos blancos los que mayor cantidad de gas producen frente a los tintos.

Esta diferencia de producción de gas entre orujos blancos y tintos puede ser debido a una mayor presencia de carbohidratos fácilmente fermentables en los orujos blancos, ya que, en la elaboración de vinos blancos, no se lleva a cabo el proceso de maceración, por tanto, el orujo es retirado tras el prensado o estrujado, conservando un mayor nivel de fructosa (Deng, Penner \& Zhao 2011). De acuerdo con Torres-Salado

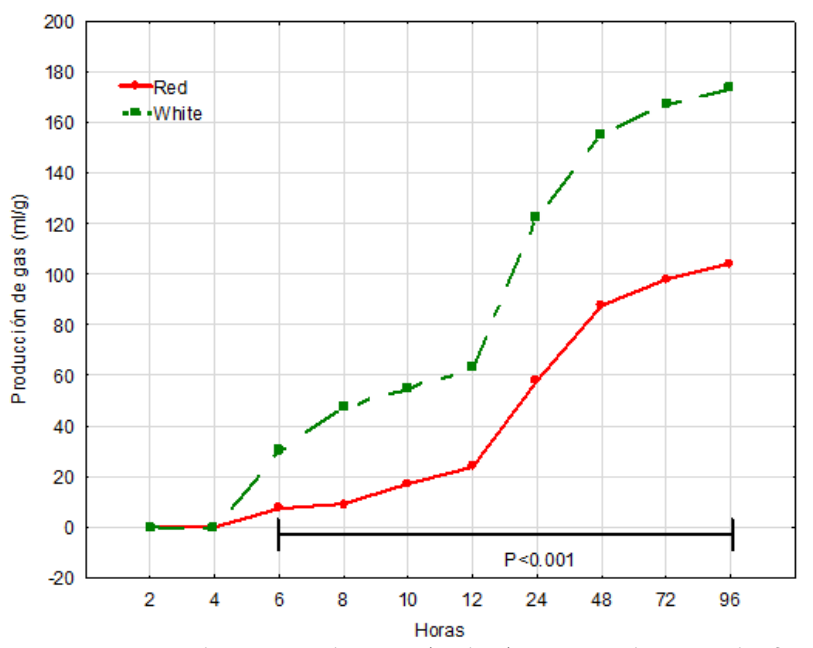

Figura 1. Producción de gas ( $\mathrm{ml} / \mathrm{g})$ tras 96 horas de fermentación (Gas production (ml/g) after 96 hours of fermentation).

Tabla I. Composición química de los orujos de estudio, expresado en $\mathrm{g} / \mathrm{kg}$ materia seca (Chemical composition of study orujos, expressed in $\mathrm{g} / \mathrm{kg}$ dry matter).

\begin{tabular}{|c|c|c|c|c|c|c|c|}
\hline \multirow[t]{2}{*}{ VARIEDAD } & EST. & M.O & G.B & P.B & F.B & F.N.D & F.A.D \\
\hline & $\mathrm{P}$ & 0.560 & 0.173 & 0.307 & 0.055 & 0.222 & 0.424 \\
\hline \multirow[t]{5}{*}{ OR.B } & Med & 926.7 & 66.6 & 112.4 & 266 & 541.7 & 468.2 \\
\hline & D.T & 6.9 & 17.4 & 4.9 & 32.9 & 56 & 42 \\
\hline & C.V & 0.75 & 26.15 & 4.40 & 12.35 & 10.35 & 8.97 \\
\hline & Max. & 935.6 & 91.2 & 118.4 & 309.9 & 610.3 & 508.5 \\
\hline & Min. & 916.4 & 48.2 & 105.3 & 205.1 & 462.6 & 404.6 \\
\hline \multirow[t]{5}{*}{ OR.T } & Med & 928.6 & 54.6 & 105.7 & 236.5 & 498.7 & 444.7 \\
\hline & D.T & 5.2 & 14.4 & 17.0 & 17.7 & 73.8 & 67.4 \\
\hline & C.V & 0.005 & 0.26 & 0.16 & 0.07 & 0.14 & 0.15 \\
\hline & Max. & 936.0 & 75.0 & 132.5 & 259 & 654.1 & 587.2 \\
\hline & Min. & 922.5 & 32 & 81.7 & 203.8 & 440.9 & 381.4 \\
\hline \multirow[t]{5}{*}{ TODOS } & Med & 927.6 & 61 & 109.3 & 252.2 & 521.6 & 457.3 \\
\hline & D.T & 6.1 & 16.7 & 12.2 & 30.1 & 66.3 & 54.5 \\
\hline & C.V & 0.006 & 0.27 & 0.11 & 0.11 & 0.12 & 0.11 \\
\hline & Max. & 936 & 91.2 & 132.5 & 309.9 & 654.1 & 587.2 \\
\hline & Min. & 916.4 & 32.0 & 81.7 & 203.8 & 440.9 & 381.4 \\
\hline
\end{tabular}

EST: estadísticos; M.O: materia orgánica; G.B: grasa bruta; P.B: proteína bruta; F.B: fibra bruta; F.N.D: fibra neutro detergente; F.A.D: fibra ácido detergente; OR.B: orujos variedad blanca; OR.T: orujos variedad tinta; P: p valor; MED: media; D.T: desviación típica; C.V: coeficiente de variación; MAX: máximo; MIN: mínimo. 
et al. (2018) en un proceso fermentativo, primero se fermentan los azúcares solubles, seguidos por polisacáridos de pared celular y, en último lugar, polisacáridos de pared lignificados. Esta hipótesis puede ser avalada por los resultados obtenidos para el parámetro " $t l$ " de la ecuación de McDonald (1981) (Tabla IV), el cual, hace referencia al tiempo de retraso del inicio de la fermentación, que a su vez varía en función del sustrato degradado y la presencia de carbohidratos solubles, (Coblentz et al. 2013). Teniendo esto en cuenta y viendo los resultados de nuestro estudio, observamos que los orujos blancos presentan un valor de " $t l$ " significativamente más bajo que los orujos tintos. Russo et al. (2017), estudiaron la producción de gas de diferentes variedades de orujos de uva (tintos y blancos), obtuvieron resultados acordes con los nuestros, siendo los orujos tintos los que menor cantidad de gas producían frente a los blancos.

Las diferencias en cuanto a la producción de gas encontrada por los diferentes autores para el orujo de uva, pueden ser debido a que los componentes que lo conforman no siempre se encuentran en la misma proporción, cada uno de ellos presenta diferencias en cuanto al contenido en fibra y perfil lipídico, influyendo directamente sobre las propiedades fermentativas, y por tanto, alterando de un modo u otro la producción de gas y $\mathrm{CH}_{4}$ en función de la cantidad presente de cada componente (Russo et al. 2017).

Además de la diferente composición química del orujo, otra causa puede asociarse a la presencia de taninos que inhiben la fermentación. Los orujos blancos no tienen transferencia al mosto de taninos por falta de fermentación conjunta con los hollejos (Gomez et al. 2007) lo que haría pensar en una mayor repercusión sobre la fermentación, a pesar de ello, los mayores niveles de polifenoles totales de las variedades tintas tanto en uvas (Gougoulias et al. 2018) como en orujo (Sagdic et al. 2011) hace que tengan una mayor actividad antimicrobiana (Kiraly et al. 2009).

En cuanto a los valores de producción de $\mathrm{CH}_{4}$ medidos a 18 y 96 horas quedan recogidos en la Tabla II y III respectivamente, podemos observar diferencias estadísticamente significativas $(\mathrm{p}<0.0001)$, siendo los orujos tintos los que menor cantidad de $\mathrm{CH}_{4}$ producen en ambos casos.

Nuestros resultados difieren de los encontrados por Russo et al. (2017) quienes no encuentran diferencias en la producción de $\mathrm{CH}_{4}$ en función del tipo de orujo utilizado. Sin embargo, están en línea con los descritos

Tabla II. Producción de gas $(\mathrm{ml} / \mathrm{g})$ y $\mathrm{CH}_{4}\left(\mathrm{mlCH}_{4} / \mathrm{g}\right)$ tras 18 horas de fermentación (Gas production $(\mathrm{ml} / \mathrm{g}$ ) and $\mathrm{CH}_{4}\left(\mathrm{mlCH}_{4} / \mathrm{g}\right)$ after 18 hours of fermentation).

\begin{tabular}{cccccc}
\hline \multicolumn{4}{c}{ OR.B } & \multicolumn{2}{c}{ OR.T } \\
\hline & Med & D.T & Med & D.T & P \\
\hline P.G 18h & 94.44 & 17.12 & 59.77 & 25.01 & $<0.0001$ \\
CH $_{4}-18 \mathrm{~h}$ & 9,66 & 2,87 & 3,72 & 1,47 & $<0.0001$ \\
\hline
\end{tabular}

OR.B: orujos variedad blanca; OR.T: orujos variedad tinta; MED: media; D.T: desviación típica; P: p valor; P.G: producción de gas. por Jayanegara, Leiber \& Kreuzer (2012) quienes en un meta-analysis de los resultados publicados sobre rumiantes, observan un claro efecto antimetanogénico en función del nivel de taninos del alimento.

Dicho efecto puede ser debido bien a la concentración de polifenoles presentes en el mismo o bien a la naturaleza de los mismos (Patra et al. 2017). A ello hay que añadir el efecto maceración, en el caso de las uvas tintas, con transferencia de polifenoles al vino (Lingua et al. 2016), por lo que se evidencia un efecto cualitativo intrínseco, vinculado a las uvas tintas.

La modelización de la producción de gas se realizó siguiendo los modelos propuestos por Orskov \& McDonald (1979) y McDonald (1981). Los resultados encontrados para los parámetros de ambas ecuaciones se recogen en la Tabla IV.

El valor de " $a$ " se encuentra vinculado a la fracción inmediatamente degradable del alimento (Orskov \&

\begin{tabular}{|c|c|c|c|c|c|}
\hline & & & & & \\
\hline & Med & D.T & Med & D.T & $P$ \\
\hline P.G 2h & 0 & 0 & 0 & 0 & n.s \\
\hline P.G 4h & 0 & 0 & 0 & 0 & n.s \\
\hline P.G 6h & 29.80 & 22.37 & 7.35 & 11.18 & $<0.0001$ \\
\hline P.G 8h & 48.07 & 31.20 & 9.24 & 12.37 & $<0.0001$ \\
\hline P.G 10h & 54.33 & 29.12 & 17.10 & 12.55 & $<0.0001$ \\
\hline P.G $12 \mathrm{~h}$ & 63.18 & 26.44 & 23.73 & 11.02 & $<0.0001$ \\
\hline P.G 24h & 122.53 & 36.34 & 57.84 & 12.08 & $<0.0001$ \\
\hline P.G 48h & 154.65 & 33.67 & 87.63 & 16.87 & $<0.0001$ \\
\hline P.G 72h & 167.64 & 32.70 & 97.94 & 14.90 & $<0.0001$ \\
\hline P.G 96h & 173.23 & 32.69 & 104.28 & 12.70 & $<0.0001$ \\
\hline $\mathrm{CH}_{4}-96 \mathrm{~h}$ & 26.08 & 8.35 & 6.79 & 1.77 & $<0.0001$ \\
\hline
\end{tabular}

OR.B: orujos variedad blanca; OR.T: orujos variedad tinta; MED: media; D.T: desviación típica; P: p valor; P.G: producción de gas.

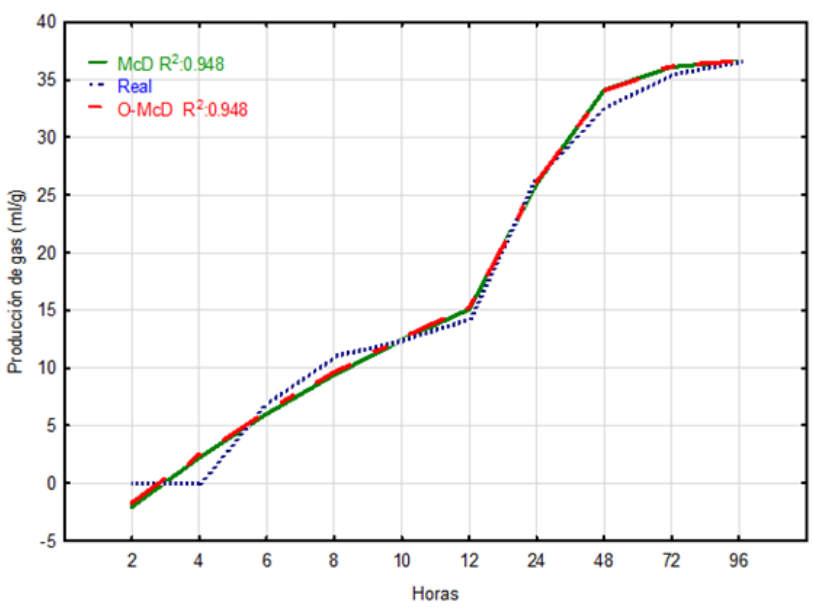

Figura 2. Modelización de producción de gas Orskov \& McDonald (1979) y McDonald (1981) para orujos blancos (Modelling of gas production Orskov \& McDonald (1979,) and McDonald (1981) for white orbs). 
Tabla IV. Modelización de la producción de gas, parámetros de la ecuación de Orskov \& McDonald (1979) y McDonald (1981) (Modelling gas production, parameters of the Orskov \& McDonald equation (1979) and McDonald (1981).

\begin{tabular}{clcccccccc}
\hline & \multicolumn{3}{c}{ Orskov } & \multicolumn{1}{c}{ McDonald (1979) } & \multicolumn{3}{c}{ McDonald (1981) } \\
& & $\mathrm{a}$ & $\mathrm{b}$ & $\mathrm{c}$ & $\mathrm{a}$ & $\mathrm{b}$ & $\mathrm{c}$ & $\mathrm{tl}$ \\
& $\mathrm{P}$ & $<0.0001$ & $<0.0001$ & $<0.0001$ & 0.178 & $<0.0001$ & $<0.0001$ & $<0.0001$ \\
\multirow{2}{*}{ OR.B } & Med & -6.45 & 43.22 & 0.06 & -0.85 & 37.62 & 0.06 & 2.54 \\
& D.T & 2.09 & 6.12 & 0.02 & 2.63 & 5.88 & 0.02 & 1.20 \\
\multirow{2}{*}{ OR.T } & Med & -3.27 & 26.96 & 0.03 & -0.13 & 23.82 & 0.03 & 4.03 \\
& D.T & 0.77 & 2.68 & 0.01 & 0.79 & 2.34 & 0.01 & 1.57 \\
\hline
\end{tabular}

A: fracción soluble o degradable a tiempo 0; b: fracción insoluble pero potencialmente degradable; c: velocidad o tasa de degradación; tl: tiempo de colonización; P: p valor; MED: media; D.T: desviación típica; OR.B: orujos variedad blanca; OR.T: orujos variedad tinta.

McDonald, 1970), presenta un valor negativo en ambas ecuaciones y para ambos orujos, al faltar la restricción de no negatividad en la resolución del problema. Un comportamiento similar en el ajuste de las ecuaciones ha sido indicado por otros autores que trabajan con pastos de baja calidad y ricos en polifenoles (Kazahaal et al. 1994) y sería indicativo de una dificultad en el inicio del proceso fermentativo, estos resultados están acordes con la dinámica real observada, puesto que hasta las 6 horas no se observa producción de gas (Figuras 2 y 3, Tabla II).

El parámetro " $c$ ", es un coeficiente de degradabilidad intrínseco o característico de cada material. En nuestro caso, encontramos el mismo valor del parámetro para ambas ecuaciones. Siendo superior en el caso del orujo blanco, probablemente debido a la presencia de un mayor nivel de carbohidratos no fibrosos y más fermentables (Basalan et al. 2011). Estos valores se aproximan a lo publicado por Alipour \& Rouzbehan (2007) para subproducto de uvas, y está acorde con el resultado real, al ser el orujo blanco más activo en la producción de gas.

Las Figuras 2 y 3 muestran los valores de cinética de producción de gas a partir de las ecuaciones propuestas, para orujos blancos y tintos respectivamente. En general, el modelo presenta una tendencia creciente

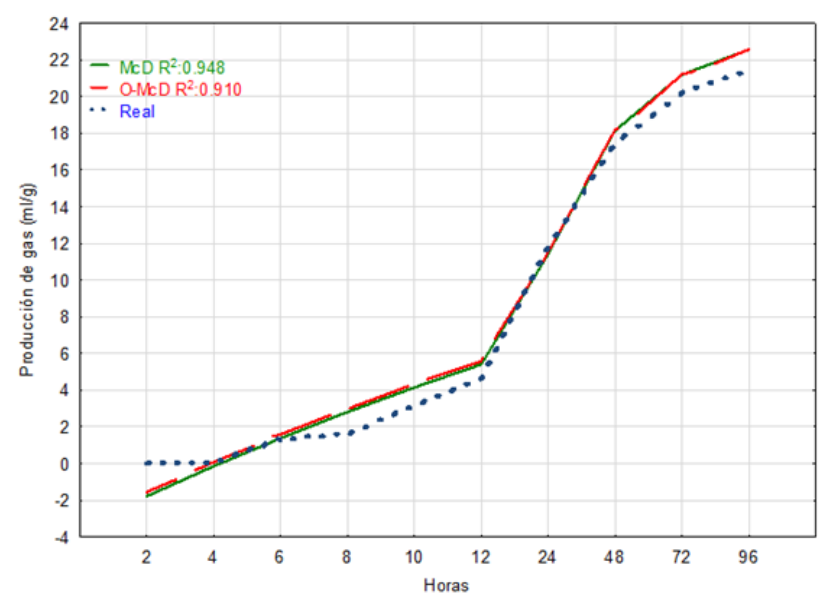

Figura 3. Modelización de la producción de gas Orskov \& McDonald (1979) y McDonald (1981) para orujos tintos (Modelling the production of Orskov \& McDonald (1979) and McDonald (1981) gas for red orbs). hasta las 48 horas de fermentación, a partir de la cual, presenta un comportamiento asintótico. Ambos modelos presentan un ajuste similar siendo en el caso de los orujos tintos el modelo de McDonald el que mejor se ajusta. El valor final de gas acumulado se asemeja al valor determinado de manera experimental, siendo prácticamente igual en el caso de los orujos tintos.

En relación al valor nutritivo, Tobias et al. (2010) realizaron un análisis de distintos vegetales, obteniendo valores de producción de gas para el tomate, jengibre y espinaca similares a los obtenidos para los orujos de estudio (Figuras 2 y 3), lo cual, es indicativo de un potencial nutritivo similar para estos productos.

\section{CONCLUSIÓN}

A vista de los resultados obtenidos, podemos concluir que los orujos tintos presentan un potencial de inhibición de producción de gas y $\mathrm{CH}_{4}$, in vitro, superior a la de los orujos blancos.

\section{AGRADECIMIENTOS}

Este trabajo ha sido financiado a través de la beca INIA CPD2016-0037. Agradecemos a Mª Jesús Guerrero, Yolanda Jiménez, J. Antonio Megías su inestimable colaboración en la realización de este trabajo, con su actividad en el laboratorio y en el manejo del ganado.

\section{BIBLIOGRAFÍA}

Abarghuei, MJ, Rouzbehan, Y, Alipour, D 2010, 'The influence of the grape pomace on the ruminal parameters of sheep', Livestock Science, vol. 132, pp. 73-79.

Aghajanzadeh, A, Maheri, N, Salmat, R, Ebrahimnezhad, Y, Ghorbani, A 2015, 'Developing a modified in vitro gas production technique to replace nylon bag method of evaluating protein degradation of alfalfa hay in ruminants', Iranian Journal of Applied Animal Science, vol. 5, no. 2, pp. 339345.

Alayón, JA, Jiménez, G, Piñeiro, AT, Canul, J, Albores, S, Villanueva, G, Nahed, J, Ku, JC 2018, 'Estrategias de mitigación de gases de efecto invernadero en la ganadería', Agroproductividad, vol. 11, no. 2, pp. 915.

Alejos, Jl, Almaraz, I, Peralta, JJG, Sánchez, P, Soriano, R, Torres, MG 2018 , 'Consideraciones de bienestar animal en las estrategias para la disminución de la producción de metano entérico por rumiantes', Agroproductividad, vol. 11, pp. 5763. 
Alipour, D \& Rouzbehan, Y 2007, 'Effects of ensiling grape pomace and addition of polyethylene glycol on in vitro gas production and microbial biomass yield', Animal Feed Science and Technology, vol. 137, pp. 138149.

Basalan, M, Gungor, T, Owens, FN, Yacinkaya, I 2011 , 'Nutrient content and in vitro digestibility of Turkish grape pomaces', Animal Feed Science and Technology, vol. 169, pp. 194198.

Baumgärtel, T, Kluth, H, Epperlein, K, Rodehutscord, M 2017, 'A note on digestibility and energy value for sheep of different grape pomace', Small Ruminant Research, vol. 67, pp. 302-306.

Broucek, J 2018, 'Methane abatement strategies based on genetics and dietary manipulation of ruminants: a review', Archivos de Zootecnia, 67(259), pp. 448458.

Coblentz, WK, Nellis, SE, Hoffman, PC, Hall, MB, Weimer, PJ, Esser, NM, Bertram, MG 2013, 'Unique interrelationships between fiber composition, water-soluble carbohidrates, and in vitro gas production for allgrown oat forages', Journal of Dairy Science, vol. 96, pp. 71957209.

Damiran, D \& Yu, P 2010, 'Chemical profile, rumen degradation kinetics, and energy value of four hullless barley cultivars: comparison of the zeroamylose waxy, waxy, highamylose, and normal starch cultivars', Journal of Agricultural and Food Chemistry, vol. 58, no. 19, pp. 10553-10559.

Deng, Q, Penner, MH, Zhao, Y 2011 , 'Chemical composition of dietary fiber and polyphenols of five different varieties of wine grape pomace skins', Food Research International, vol. 44, pp. 2712-2720.

EC (2003) Regulation (EC) No 1831/2003 of the European Parliament and of the Council of 22 September 2003 on additives for use in animal nutrition (Text with EEA relevance). Official Journal of the European Union, 18102003.

EC (2009) Commission Regulation (EC) No 152/2009 of 27 January 2009 laying down the methods of sampling and analysis for the official control of feed. Official Journal of the European Union, 26022009.

ElMeadaway, A, Mir, Z, Mir, PS, Zaman, MS, Yanke, L 1998, 'Relative efficacy of inocula from rumen fluid or faecal solution for determining in vitro digestibility and gas production', Canadian Journal of Animal Science, vol. 78, no. 4, pp. 673679

El Shaer, HM, Omed, HM, Chamberlain, AG 1987, 'Use of faecal organisms from sheep for the in vitro determination of digestibility', Journal of Agricultural Science Cabrigde, vol. 109, pp. 257259.

Foiklang, S, Wanapat, M, Norrapoke, T 2016, 'In vitro rumen fermentation and digestibility of buffaloes as influenced by grape pomace powder and urea treated rice straw supplementation', Animal Science Journal, vol. 87, pp. 370-377.

Gómez, MJ, González, ML, Hernanz, D, Fernández, MA, Vicario, IM, Heredia, FJ 2007, 'Effects of prefermentative skin contact conditions on colour and phenolic content of white wines', Journal of Food Engineering, vol. 78, pp. 238-245.

Gougoulias, N, Vagelas, I, Giurgiulescu, L, Ntalla, MN, Nterkou, P, Salonikioti, A, Efthimionu, S, Sugar, IR 2018, 'Comparative study on polyphenols content and antioxidant effect of some grape varieties grown in central Greece', Carpathian Journal of Food Science and Technology, vol. 10, no. 1, pp. 141149.

Hristov, AN, Oh, J, Giallongo, F, Frederick, TW, Harper, MT, Weeks, HL, Branco, AF, Moate, PJ, Deighton, MH, Williams, SRO, Kindermann, M, Duval, S 2015, 'An inhibitor persistently decreased enteric methane emission from dairy cows with no negative effect on milk production', Proceedings of the National Academy of Sciences of the United States of America, vol. 112, no. 34, pp. 10663-10668.

Hook, SE, Wright, ADG, McBride, BW 2010, 'Methanogens: methane producers of the rumen and mitigation strategies', Archaea vol. 2010, pp. 111

IPCC (2007). Cambio climático 2007. Base de Ciencia Física. Grupo de Trabajo I. Cuarto Informe de Evaluación del Panel Intergubernamental sobre Cambio Climático. Resumen. https://www.ipcc.ch/site/assets/ uploads/2020/02/ar4wg 1 sumvolsp.pdf
Jayanegara, A, Leiber, F, Kreuzer, M 2012, 'Metaanalysis of the relationship between dietary tannin level and methane formation in ruminants from in vivo and in vitro experiments', Journal of Animal Physiology and Animal Nutrition, vol. 96, pp. 365375.

Kiraly, Z, Moricz, AM, Ott, PG, Katay, G, Belai, I, Tyihak, E 2009, 'Comparison of components from red and white wines for antimicrobial activity by biodetection after OPLC separation', Journal of Liquid Chromatography \& Related Technologies, vol. 32(9), pp. 12591272.

Lingua, MS, Fabani, MP, Wunderlin, DA, Varoni, MV 2016, 'From grape to wine: Changes in phenolic composition and its influence on antioxidant activity', Food Chemistry, vol. 208, pp. 228238.

Marginet, X 2004, project Life 04 ENV/ES/000237, 'Grape Tannins Saving of forest exploitation for obtaining of tanning extracts through valorisation of wine waste', https://ec.europa.eu/environment/life/ project/Projects/index.cfm? fuseaction=search.dspPage\&n_proj_ id=2687\&docType=pdf Last consult Febraury 2020.

McDonald, I 1981, 'A revised model for the estimation of protein degradability in the rumen', Journal of Agricultural Science, vol. 96, no. 1, pp. 251252.

McDonald, P, Edwards, RA, Greenhalgh, JFD, Morgan, CA, Sinclair, LA, Wilkinson, RG 2010, animal nutrition, $7^{\text {th }}$ edition (Chp. 8), Pearson, Harlow, England, U.K.

McSweeney, CS, Palmer, B, McNeill, DM, Krause, DO 2001, 'Microbial interactions with tannins: nutritional consequences for ruminants', Animal Feed Science and Technology, vol. 91, pp. 8393.

Menke, KH, Raab, L, Salewski, A, Steingass, H, Fritz, D, Schneider, W 1979, 'The estimation of digestibility and metabolizable energy content of ruminants feedstuffs from the gas production when they are incubated with rumen liquor in vitro', Journal of Agricultural Science Cambrigde, vol. 93, no. 1, pp. 217222.

Moate, P J, Williams, SRO, Torok, VA, Hannah, MC, Ribaux, BE, Tavendale, MH, Eckard, RJ, Jacobs, JL, Auldist, MJ, Wales, WJ 2014, 'Grape marc reduces methane emissions when fed to dairy cows', Journal Dairy Science, vol. 97, pp. 50735087.

Moumen, A, Azizi, G, Chekroun, K, Ben, Baghour, M 2016, 'The effects of livestock methane emission on the global warming: A review', International Journal of Global Warming, vol. 9, no. 2, pp. 229-253.

Ørskov, ER \& McDonald, IM 1979, 'The estimation of protein degradability in the rumen from incubation measurements weighted according to rate of passage', Journal of Agricultural Science Cambridge, vol. 92, pp. 499503.

Patra, AK \& Saxena, J 2010, 'A new perspective on the use of plant secondary metabolites to inhibit methanogenesis in the rumen', Phytochemistry, vol. 71, no. 1112, pp. 11981222.

Patra, A, Park, T, Kim, M, Zhongtang, Y 2017, 'Rumen methanogens and mitigation of methane emission by antimethanogenic compounds and substances', Journal of Animal Science and Biotechnology, vol. 8, pp. 1331.

Peixoto, CM, Dias, MI, Alves, MJ, Calhelha, RC, Barros, L, Pinho, SP, Ferreira, I 2018, 'Grape pomace as a source of phenolic compounds and diverse bioactive properties', Food Chemistry, vol. 253, pp. 132138.

Ramanathan, V \& Feng, Y 2009, 'Air pollution, greenhouse gases and climate change: Global and regional perspectives', Atmospheric Environment, vol. 43, pp. 37-50.

Rasoul, B \& Amiri, B 2016, `Nutritive Value Assessment of Three Rangeland Species Using Sheep Rumen and Feces Fluid', Environmental Resources Research, vol. 4, no.2, pp.141152.

Russo, VM, Jacobs, JL, Hannah, MC, Moate, PJ, Dunshea, FT, Leury, BJ 2017, 'In vitro evaluation of the methane mitigation potential of a range of grape marc products', Animal Production Science, vol. 57 , no. 7, pp. 14371444.

Sagdic, O, Ozturk, I, Ozkan, G, Yetim, H, Ekici, L, Yilmaz, MT 2011, 'RPHPLC-DAD analysis of phenolic compounds in pomace extracts from five grape cultivars: Evaluation of their Antioxidant, antiradical and antifungal activities in orange and apple juices', Food Chemistry, vol. 126, pp. 17491758. 
Salado, N, Santillán, P, García, A.R., Pérez, J., Morales, J. (2018). Producción de gases efecto invernadero in vitro de leguminosas arbóreas del trópico seco mexicano. Archivos de Zootecnia, 67 (257), pp. 5559.

Steinfeld, H, Gerber, P, Wassenaar, T, Castel, V, Rosales, M, De Haan, C 2006, Livestock's Long Shadow: Environmental Issues and Options, FAO, Rome, Italy.
Tobias, C, Hector, B, Mazza, PH, Oliveira, LM, Marques, P, Alves, EJ, Ørskov, ER 2010, 'Characterization of vegetables and fruits potential as ruminant feed by in vitro gas production technique', Livestock Research for Rural Development, vol. 22, no. 9, Art, 168.

Van Soest, PJ 1967, 'Development of a comprehensive system of feed analyses and its application to forages', Journal of Animal Science, vol. 26, no. 1, pp. 119128 . 